\title{
Haemorrhagic stroke as the first presentation of undiagnosed infective endocarditis in an 8 year old boy
}

\author{
*Gurmeet Singh ${ }^{1}$, Sunita Arora ${ }^{2}$, Gursharan Singh Narang ${ }^{2}$ \\ Sri Lanka Journal of Child Health, 2015; 44(2): 114-116
}

(Keywords: Infective endocarditis, mycotic aneurysm, cerebral haemorrhage)

\section{Introduction}

Infective endocarditis (IE) is uncommon in children with an incidence of around $0.6 / 100,000$ /year but causes a mortality of $11-20 \%{ }^{1}$. Only $7 \%$ of children having IE had neurological complications ${ }^{1}$. Ruptured mycotic aneurysm accounts for only 5\% of neurological complications of $\mathrm{IE}^{2}$. Rarely a ruptured mycotic aneurysm can be the first manifestation of IE. This catastrophic event is associated with $80 \%$ mortality $^{3}$. We present an 8 year old boy who presented with haemorrhagic stroke but was later confirmed to have features of mitral regurgitation along with mobile vegetations on anterior mitral leaflet.

\section{Case report}

An 8 year old boy presented with non-projectile, non-bilious vomiting for two days associated with weakness of left upper and lower limbs and loss of consciousness lasting about 12 hours. There was no history of fever, seizures, ear discharge, rash, respiratory illness, headache, photophobia, decreased vision, mucocutaneous bleeding or trauma. Child was irritable and disoriented at the time of presentation. On central nervous system (CNS) examination, he had left sided facial paralysis and hypotonia of left upper and lower limbs. Power was $2 / 5$ in left upper and lower limbs. Deep tendon reflexes were exaggerated with extensor plantar response on the left side. Meningeal signs were present. A grade 4 systolic murmur was detected in the mitral area with selective propagation to the axilla. No bruit was audible over the cranium or carotid arteries. Child was afebrile and normotensive.

Computed tomography (CT) scan revealed an intraparenchymal bleed on the right side about $5.1 \times 2.0 \mathrm{~cm}$ with intraventricular extension and midline shift (Figure 1).

${ }^{1}$ Associate Professor, ${ }^{2}$ Professor, Department of Paediatrics, Sh. Guru Ram Das Institute of Medical Science \& Research, Amritsar, India

*Correspondence:dr.gmsingh@gmail.com

(Received on 7 April 2014: Accepted after revision on 30 May 2014)

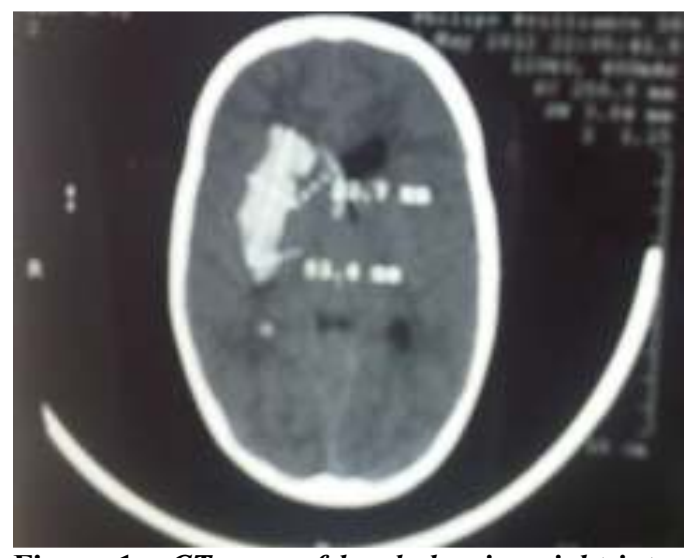

Figure 1: CT scan of head showing right intraparenchymal bleed

Complete blood count showed polymorphonuclear leucocytosis with normal bleeding and coagulation profile. CT angiogram revealed a narrow neck $(0.6 \mathrm{~mm})$ saccular out-pouching measuring about $6.4 \times 4.8 \mathrm{~cm}$ in the region of the M2 (sylvian) segment of right middle cerebral artery (MCA) with right ganglio-capsular bleed and midline shift towards the left (Figure 2).

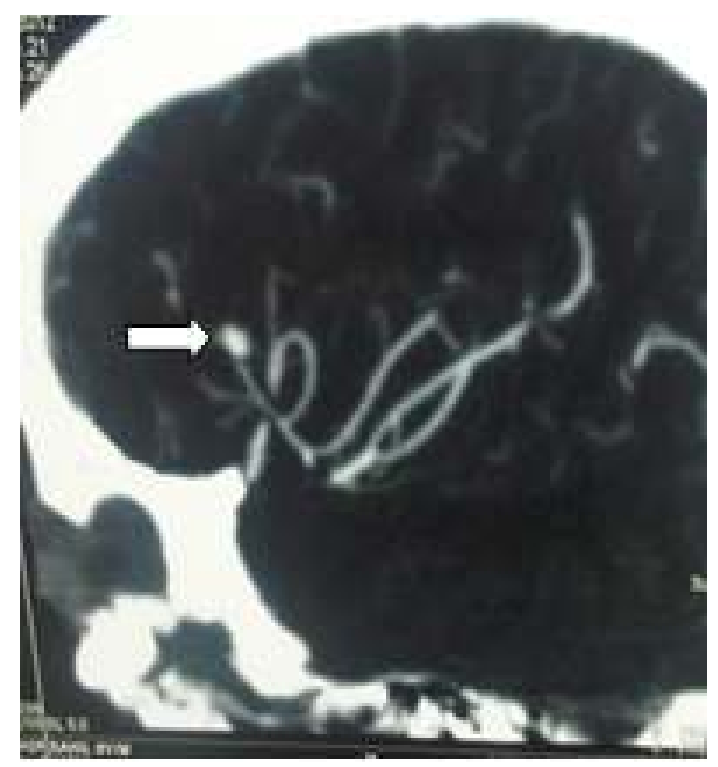

Figure 2: CT angiogram showing saccular aneurysm 
Echocardiography revealed severe mitral valve insufficiency with a $5 \mathrm{~mm}$ mobile vegetation on anterior mitral leaflet suggestive of infective endocarditis. Though three consecutive blood cultures were sterile, acute phase markers such as C-reactive protein (CRP) and erythrocyte sedimentation rate (ESR) were raised. Child was empirically started on antibiotic therapy for endocarditis and surgical clipping of neck of aneurysm was done after 72 hours. Postoperative period was uneventful. Child gradually improved and was able to walk with a limp after about 2-3 weeks.

\section{Discussion}

Saccular or congenital aneurysms constitute $90 \%$ of all cerebral aneurysms and are located at the major branch points of the large arteries ${ }^{4}$. Fusiform aneurysms are elongated outpouchings of proximal arteries that account for $7 \%$ of all cerebral aneurysms. Mycotic aneurysms are situated peripherally and comprise $0.5 \%$ of all cerebral aneurysms ${ }^{4}$. Saccular aneurysms are rare in the paediatric population ${ }^{4}$. No underlying systemic disorder is detected in the majority. One third to one half of paediatric aneurysms arise from the posterior circulation ${ }^{4}$. Conditions which are associated with paediatric cerebral aneurysms include: polycystic kidney disease, coarctation of aorta, tuberous sclerosis, alpha-glucosidase deficiency, alpha-1-antitrypsin deficiency and Parry-Romberg syndrome ${ }^{5}$.

Intracranial aneurysms often present with signs and symptoms of subarachnoid haemorrhage, including severe headache, vomiting, and obtundation, which may progress to coma, or recurrent headaches ${ }^{5}$. Giant aneurysms (diameter greater than $2.5 \mathrm{~cm}$ ) are found in $20-40 \%$ of affected paediatric patients and may present with focal neurological symptoms and signs due to compression of the surrounding brain ${ }^{5}$. Angiography is essential for anatomical details like location and size of the neck and sac and to locate additional aneurysms ${ }^{5}$.

Mycotic aneurysms result from any infectious process of bacterial, fungal, and protozoan origin ${ }^{6}$. The most common cause of mycotic aneurysms is an underlying bacterial endocarditis from which infectious thrombi are embolized into the intracranial circulation. The acute inflammation leads to neutrophilic infiltration followed by degradation of the media and adventitia, fragmentation of the internal elastic lamina and proliferation of the intima. The weakened vessel wall in combination with the pulsatile pressure in the vasculature leads to aneurysm formation and consequential growth ${ }^{6}$. Mycotic aneurysms were detected in $2 \%$ of cases of endocarditis, comparable to rates reported in studies that have incorporated both adult and paediatric patients ${ }^{7}$. Some of the findings on angiography that point toward mycotic aneurysms are the fusiform shape, the multiplicity, the distal location, and the change in size on follow-up angiography. Positive culture from the wall itself can confirm the diagnosis. Other indicators are positive blood culture (only found in $35.6 \%$ ), leucocytosis, elevated ESR, and elevated $\mathrm{CRP}^{6}$. However, saccular morphology does not exclude it, as it has been shown that approximately $41 \%$ of mycotic aneurysms reported in the literature are saccular ${ }^{6}$.

Following appropriate antimicrobial therapy, endovascular occlusion of the aneurysm or occlusion of the involved vessel segment immediately proximal to the aneurysm origin can be considered. The decision regarding when to treat a mycotic aneurysm depends upon the size and location of the aneurysm, the clinical presentation, and observed changes in the patient and the aneurysm over time ${ }^{5}$. Given that stroke occurred in only $6 \%$ of all children with endocarditis, routine surveillance screening with $\mathrm{CT}$ or MRI in all children with endocarditis cannot be advocated ${ }^{8}$.

In our case the child presented with intraparenchymal bleed from saccular aneurysms of middle cerebral artery in its sylvian segment. Mitral incompetence was incidentally detected and vegetation was detected on anterior mitral leaflet on echocardiography in the absence of any fever or constitutional symptoms or symptoms referable to cardiac lesion. The present case highlights that $\mathrm{CT} / \mathrm{MRI}$ angiography is essential in subarachnoid haemorrhage or intra-parenchymal haemorrhage to detect any aneurysm or AV malformation. Even in absence of fever and constitutional symptoms, one should search for any evidence of infective endocarditis by echocardiography and blood culture if cardiac lesion is detected clinically in the presence of haemorrhagic stroke.

\section{References}

1. Coward K, Tucker N, Darville T. Infective endocarditis in Arkansas children from 1990 through 2002. Pediatric Infectious Disease Journal 2003; 22: 1048-52. http://dx.doi.org/10.1097/01.inf.0000101186.8 8472.b5

PMid: 14688563

2. Pruit AA, Rubin RH, Karchmer AW, Duncan GW. Neurologic complications of bacterial endocarditis. Medicine 1978; 57: 329-43. http://dx.doi.org/10.1097/00005792197807000 $-00004$ 
3. Bohmfalk GL, Story JL, Wissinger JP, Brown WE. Bacterial intracranial aneurysms Journal of Neurosurgery 1978; 48: 369-82.

http://dx.doi.org/10.3171/jns.1978.48.3.0369

PMid: 580294

4. Liebeskind DS, Lutsep HL(chief editor). Cerebral Aneurysms [cited 2014 Apr 6]. Available from:

http://emedicine.medscape.com/article/116151 8-overview\#aw2aab6b2b2.

5. Endovascular Surgical Neuro Radiology [homepage on internet]. Pediatric Cerebral Aneurysms [cited 2014 Apr 6]. Available from:

http://www.esnr.com/public/studies/pediatriccerebral-aneurysms.shtml.

6. Ducruet AF, Hickman ZL, Zacharia BE, Narula R, Grobelny BT, Gorski J, et al. Intracranial infectious aneurysms: a comprehensive review. Neurosurgical Review 2010; 33(1): 37-45.

http://dx.doi.org/10.1007/s10143-009-0233-1

PMid: 19838745
7. Niwa K, Nakazawa M, Tateno S, Yoshinaga M, Terai M. Infective endocarditis in congenital heart disease: Japanese national collaboration study. Heart 2005; 91: 795-800. http://dx.doi.org/10.1136/hrt.2004.043323 PMid: 15894782

8. Venkatesan C, Wainwright MS. Pediatric endocarditis and stroke: a single-centre retrospective review of seven cases. Pediatric Neurology 2008; 38(4): 243-7. http://dx.doi.org/10.1016/j.pediatrneurol.2007. 12.009

PMid: 18358401 